\title{
Numerical analysis of energy absorption behaviour of circular profiles with plates under lateral loads
}

\author{
Quirino Estrada $^{1}$, Dariusz Szwedowicz ${ }^{2}$, Alejandro Rodriguez-Mendez ${ }^{3}$, Jesús Silva-Aceves ${ }^{1}$, Lara C. Wiebe ${ }^{1}$ \\ Javier S. Castro ${ }^{1}$, Elifalet López ${ }^{1}$, Luis F. Tinoco ${ }^{1}$ and Sebastián A. Castillo ${ }^{1}$ \\ ${ }^{1}$ Instituto de Ingeniería y Tecnología, Universidad Autónoma de Ciudad Juárez (UACJ), Ciudad Juárez, Chihuahua, México \\ ${ }^{2}$ Centro Nacional de Investigación y Desarrollo Tecnológico (CENIDET), Cuernavaca, Morelos, México \\ ${ }^{3}$ Department of Mechanical Engineering, University of California, Berkeley, CA 94720, USA
}

\begin{abstract}
The study of bending behaviour of thin-walled structures has gained importance since lateral impact is the second most common scenario in automobile crashes. The current paper analyses an effect of partition plates on energy absorption $\left(E_{a}\right)$ of circular profiles under lateral loads. For this purpose, several numerical analyses using Abaqus/Explicit finite element software were carried out. The evaluated specimens have circular cross-sections reinforced by different arrangement of partition plates. In order to get reliable outcome, special emphasis was placed on damage modelling by Johnson-Cook failure model for aluminium. From the results considering a single profile, better $E_{a}$ was registered for structures with plates in a range from $6 \%$ to $34 \%$. Reduction in peak load $\left(\mathrm{P}_{\max }\right)$ up to $13 \%$ and an increase in crush force efficiency (CFE) in $14.86 \%$ was also computed. Regarding profiles with plates, it was determined that crashworthiness performance depends on an arrangement of plates on the cross-section more than their thickness and number. Better performance was obtained when the circular cross-section was reinforced in the longitudinal and transversal direction by 4 plates.
\end{abstract}

\section{Introduction}

Every year an estimated 1.25 million deaths around the world associated to road traffic deaths are reported [1]. According to classification of crashes, collisions can occur from the frontal, rear and lateral direction. In all cases passengers receive several injuries mainly in the cervical, chest, abdominal and head areas. However, sideimpact crashes are more likely to be fatal [2]. In order to mitigate such harmful effects thin-walled structures are widely employed to absorb energy by plastic deformation. In this sense a group of structures denominated multi-cells present better energy absorption performance to be compared with single profiles [3]. Multi-cell structures are characterised by a number of plates connected with each other at various angles and with different edge connectivity. Regarding multi-cell structures subjected to lateral loads several theoretical, computational and experimental studies have been carried out. Wang et al. [4] investigated bending resistance of thin-walled multicell squares tubes. The evaluated specimens present a square cross-section reinforced by plates in the longitudinal direction. Numerical and experimental results reveal importance of the number of plates on energy absorption performance. Huang [5] studied energy absorption and optimisation design of multi-cell squared tubes. Three different cross sections were analysed and their effects on deformation process were obtained. Zhang et al. [6] carried out experimental and numerical investigation on bending collapse of embedded multi-cell tubes. The structures were obtained by embedding a group of small square tubes into an outer tube. Some of the effects, experimentally evaluated, were friction and end treatment. Regarding traditional multi-cell profiles, the embedded tubes have an increase in bending resistance of the order of $65 \%-72 \%$. Regarding studies of multi-cell structures applied to automobile designs, Ghadianlou et al. [7] presented a numerical crashworthiness design for side door beams. Several circular cross-sections reinforced with ribs were analysed. As a result, a rectangular-crossover cross-section had the best crashworthiness performance. Estrada et al. [3] evaluated an effect of the cross-section on energy absorption performance of multi-cell profiles. The numerical simulations determined effectiveness of circular multicell profiles to absorb energy by plastic deformation. Therefore, the numerical study of multi-cell profiles is a relevant topic. However, in most cases the studies have focused on square base cross-sections but not on multicell circular profiles.

Thus, in the current article energy absorption of circular multi-cell profiles is analysed. For this purpose, we carried out numerical simulations using the Abaqus finite element software. The structures studied present a single circular profile reinforced in longitudinal and transversal directions by partition plates. The assessment was carried out by quasi-static, three-point bending test and subsequent calculation of crashworthiness parameters. Numerical simulations were more realistic by incorporating a damage initiation criterion.

\subsection{Bending mechanism of single circular profiles}

According to Elchalakani et al. [8] the bending collapse mechanism of the single circular profile is characterised by formation of a central hinge line (AC) at a plastic dent depth. Likewise, the hinge lines EA and EC were observed. From these lines, a triangular shape determined by the point ACE was formed as seen in Figure 1. A

* Corresponding author: quirino.estrada@uacj.mx 
Mirror deformation pattern is observed on the opposite side of the central hinge line (AC). In this way, a star shape is formed. Regarding the cross-section, an ovalisation plateau phase was registered. This means the progressive transition from an initial circular crosssection to an oval shape.

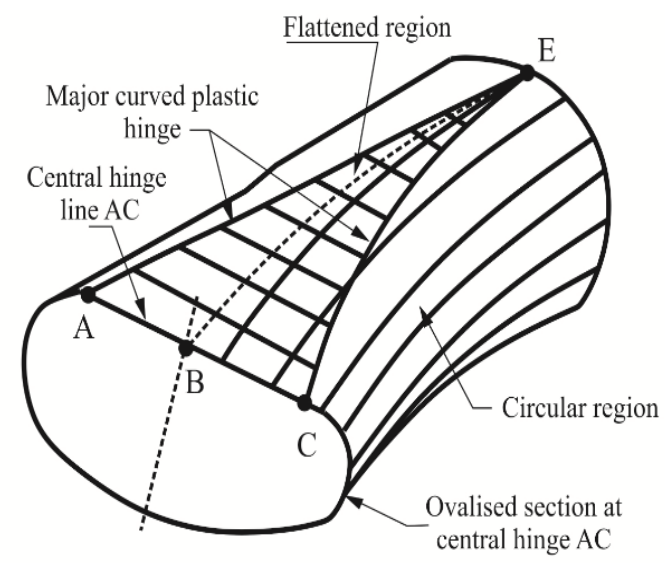

Fig. 1. Theoretical bending collapse mechanism of a circular profile [8].

\subsection{Crashworthiness indicators}

The assessment of thin-walled structures is carried out with dimensional and dimensionless parameters. Such parameter allows capturing energy absorption by elastic, plastic and frictional effects. The most important parameters are a peak load $\left(\mathrm{P}_{\max }\right)$, energy absorption $\left(\mathrm{E}_{\mathrm{a}}\right)$, mean load (Pm), crush force efficiency (CFE) and specific energy absorption (SEA), which are described in Figure 2.
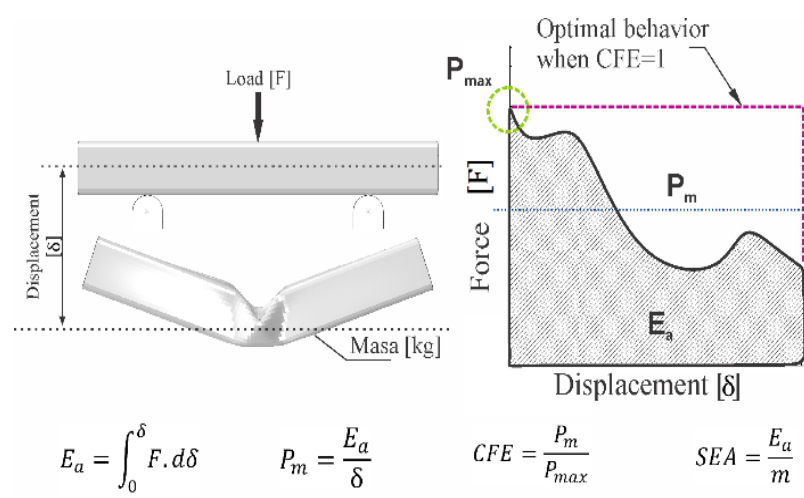

Fig. 2. Crashworthiness indicators.

\subsection{Material modelling}

During bending collapse, mechanical behaviour of the profiles becomes complex, especially when ductile materials e.g. aluminium are subjected to lateral loads. When plastic lines are formed, the structures can experience a progressive failure of the material from the elastic regime until fracture. Then, an appearance and growth of crack commence, indicating the presence of ductile and shear stress mechanisms (see Fig. 3). In this way, numerical simulations should consider theoretical failure models in order to get reliable results. For this purpose, in the current paper, the phenomenological
Johnson-Cook failure model (F J-C) is used to model progressive damage behaviour. Additionally, elasticity and plasticity models were used. The formulation for the $\mathrm{J}-\mathrm{C}$ damage model is described by Eqn. 1 [9].

\section{Ductile fracture Shear fracture}

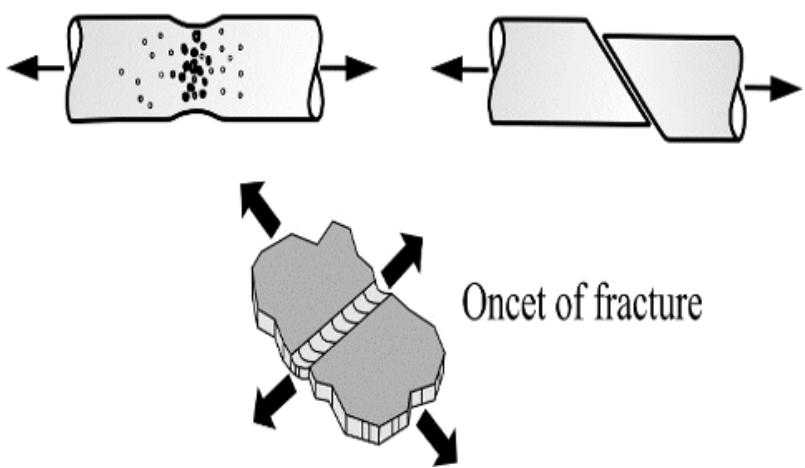

Fig. 3. Common failure mechanism in thin walled-structures, based on [10].

$\dot{\bar{\varepsilon}}^{p l}=\left[d_{1}+d_{2} \exp \left(d_{3} \frac{p}{q}\right)\right]\left[d_{4} \ln \left(\frac{\dot{\bar{\varepsilon}}^{p l}}{\dot{\varepsilon}_{0}}\right)\right]\left(1+d_{5} \hat{\theta}\right)$

where $(p)$ is a pressure-deviatoric stress ratio, $(q)$ is von Misses stress, and $(\hat{\theta})$ is non-dimensional temperature which is defined as the ratio of $\theta-\theta_{T}$ and $\theta_{m}-\theta_{t}$, where $\theta$ is current temperature, $\theta_{\text {melt }}\left[{ }^{\circ} \mathrm{K}\right]$ is melting temperature and $\theta_{t}\left[{ }^{\circ} \mathrm{K}\right]$ transition temperature. Additionally, $d_{1}-d_{5}$, are material constants. All structures analysed in the current article were made with aluminium alloy 6063 T6. The Table 1 presents mechanical properties used in the current article. A value of $126 \mathrm{MPa}$ for yield stress was used.

Table 1. Material properties for Aluminium Alloy 6063-T6 $[11,12]$.

\begin{tabular}{c|ccccc}
\hline \multirow{2}{*}{ Elast. } & E [MPa] & & Poison ratio & \multicolumn{2}{c}{ Density $\left[\mathbf{k g} / \mathbf{m}^{3}\right]$} \\
& 69000 & & 0.33 & \multicolumn{2}{c}{2700} \\
\hline \multirow{2}{*}{ F J-C } & D1 & D2 & D3 & D4 & D5 \\
& -0.77 & 1.45 & 0.47 & 0.0 & 1.6 \\
\hline
\end{tabular}

\section{First discrete model and validation}

In order to verify feasibility of obtained numerical results, a first discrete model, using Abaqus/explicit, was prepared. The model assesses bending behaviour of a circular profile by the three-point bending test of experimental analysis by Li et al. [12]. The profile was modelled using deformable shell elements (S3R), meanwhile a punch and supports were modelled as rigid bodies (R3D4). The mesh size was $2 \mathrm{~mm}$ and it was validated through experimental data obtained by Li et al. [12]. Regarding the material properties, the profile was covered with elastic, plastic and J-C damage models for aluminium alloy 6063-T6 (see Table 1). General properties such as geometry, components, boundary conditions and interactions are presented in Figure 4. 


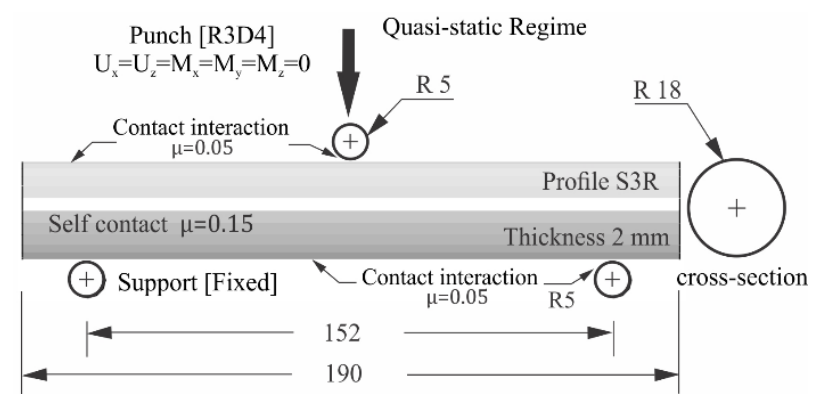

Fig. 4. General properties of the discrete model [mm].

The obtained numerical results are presented in Figures 56. Additionally, the experimental results obtained by Li et al. [12] are also shown. Figure 4 shows a typical load displacement curve which is characterised by an elastic region at the beginning of the bending process. Later, a maximum load $\left(\mathrm{P}_{\max }\right)$ of $3.2 \mathrm{~kJ}$ is reached. At this point a process of folding plastic is initiated. Subsequently, a decrease in bending load is observed. This behaviour denotes the loss of load carry capacity. Thus, effectiveness of the damage model is shown.

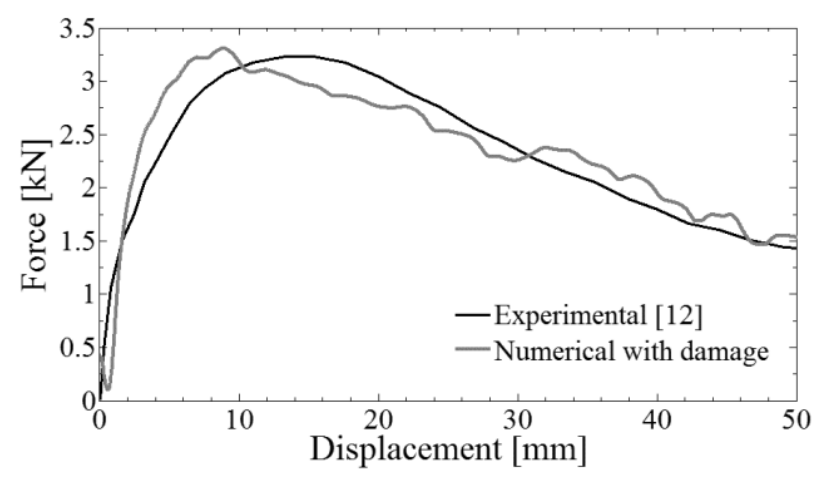

Fig. 5. Displacement-load curves for a circular profile under bending load.

The final deformation state is displayed in Figure 6. As observed, the numerical model with damage criteria correctly captures deformation of plastic, as well as the formation of travel and stationary lines.

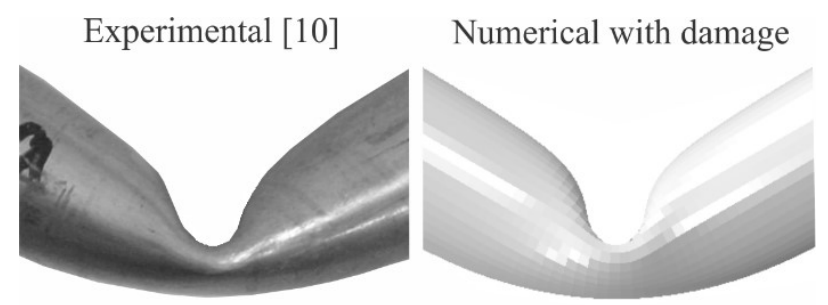

Fig. 6. Experimental vs numerical models.

A difference of $3 \%$ of $\mathrm{P}_{\max }$ was obtained between numerical and experimental results. Regarding energy absorption $\mathrm{E}_{\mathrm{a}}(2.5 \mathrm{~kJ})$, a discrepancy close to $4 \%$ was calculated. Finally, comparing the numerical results with the experimental data, feasibility of the discrete model was validated. Thus, it is possible to study multi-cell profiles by numerical techniques.

\section{Numerical simulations of multi-cell profiles}

The main objective of the current article is analysing the effect of partition plates on energy absorption of circular profiles. For this purpose, we carried out three-point bending test simulations. The multi-cell profiles were obtained through the reinforcing of single circular crosssection by partition plates in longitudinal and transversal directions (see Fig. 7). a)

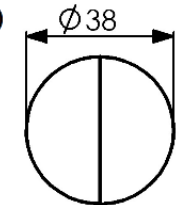

d)

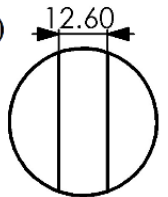

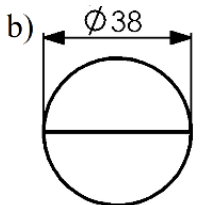

e)

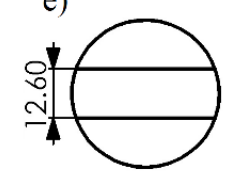

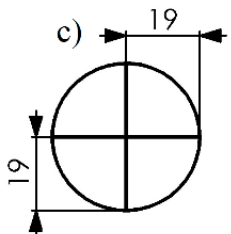

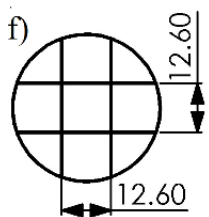

Fig. 7. Cross-sections for evaluated profiles, dimensions in $\mathrm{mm}$, where, a) MC-00, b) MC-01, c) MC-02, d) MC-03, e) MC-04, f) MC-05 and g) MC-06.

Likewise, in order to maintain the same mass, adjustments in thickness were applied. For all structures a 6063-T6 aluminium alloy and length of $190 \mathrm{~mm}$ were used. The dimensions of the evaluated specimens are presented in Table 2 .

Table 2. Dimensions of the evaluated multi-cell profiles.

\begin{tabular}{ccccc}
\hline Code & $\begin{array}{c}\text { Diameter } \\
(\mathbf{m m})\end{array}$ & $\begin{array}{c}\text { Thickness } \\
(\mathbf{m m})\end{array}$ & $\begin{array}{c}\text { Length } \\
(\mathbf{m m})\end{array}$ & $\begin{array}{c}\text { Mass } \\
(\mathbf{g r})\end{array}$ \\
\hline MC-00 & 38 & 2.65 & 190 & 162 \\
MC-01 & 38 & 2.00 & 190 & 162 \\
MC-02 & 38 & 2.00 & 190 & 162 \\
MC-03 & 38 & 1.62 & 190 & 162 \\
MC-04 & 38 & 1.65 & 190 & 162 \\
MC-05 & 38 & 1.65 & 190 & 162 \\
MC-06 & 38 & 1.20 & 190 & 162 \\
\hline
\end{tabular}

\section{Results and discussion}

The evaluation of the circular multi-cell profiles was done by the analysis of force displacement curves. Additionally, the assessment of a single circular profile is presented. In this way, a comparison baseline is obtained. Figure 8 shows the bending curves for all of the evaluated specimens. All curves are characterised by two stages, the first is related to the elastic regime, later a decrease of the bending force is observed. As a result, a maximum value of punch force $\left(\mathrm{P}_{\max }\right)$ is obtained at the beginning of the bending process followed by a drop of the load carrying capacity. In some cases, the transition between regimes was smoothed, while in other cases it was abrupt (MC-01 and MC-04). This condition is attributed to the stiffness shown by the cross-section. 


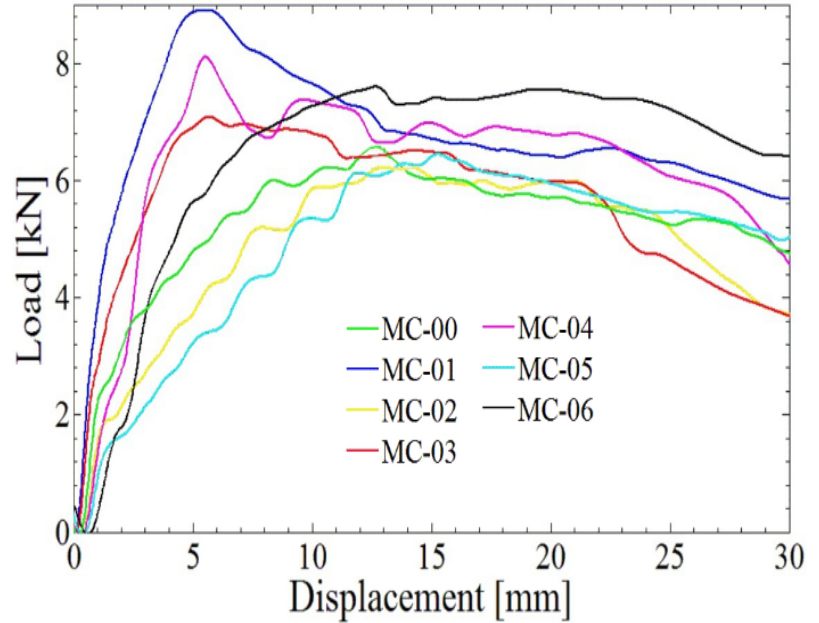

Fig. 8. Displacement-load curve for evaluated multi-cell structures.

The quantity of energy absorbed $\left(\mathrm{E}_{\mathrm{a}}\right)$ by the structures was determined using a trapezium integration method applied to the force-displacement curves and presented in Figure 9. Better $E_{a}$ performance was obtained for most multi-cell structures regarding single circular profile (MC-00). A maximum value of $0.201 \mathrm{~kJ}$ was computed for the MC-01 structure.

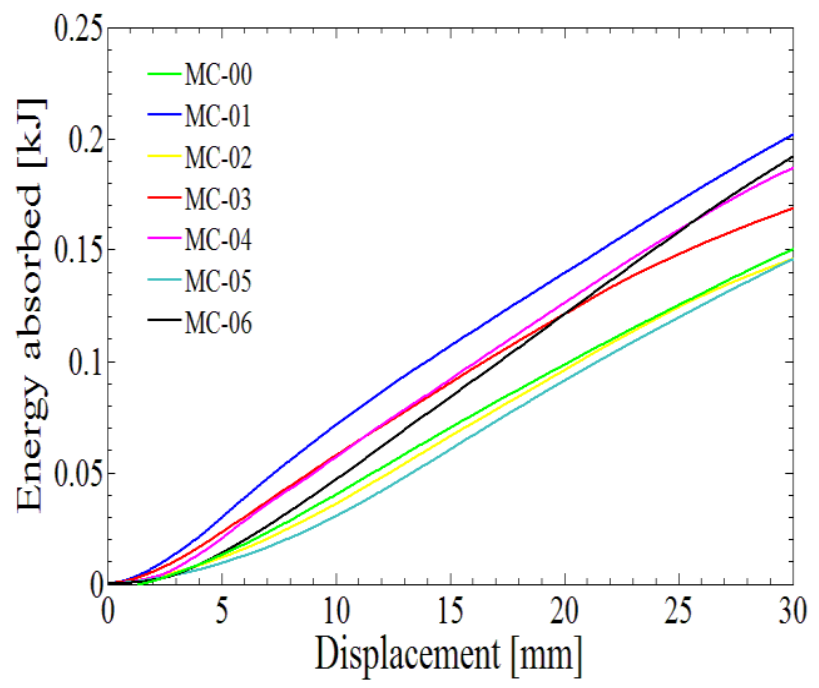

Fig. 9. Energy absorbed $\left[E_{a}\right]$ by the evaluated multi-cell structures.

Energy absorbed is directly influenced by deformation of the structures. An increase in $E_{a}$ means a resistance to the formation of stationary and traveling hinge lines. The deformation pattern was determined by stiffness of the cross-sections. Hence, a typical bending deformation mode for a single circular profile was modified (see Fig. 10-11). Thus, the ovalisation phase had some changes which in some cases led to better load carrying capacity. The failure of the structures was reached mainly by ductile effects at the upper central region of the profiles. Fracture was only observed on the MC-04 profile as seen in Figure 11.
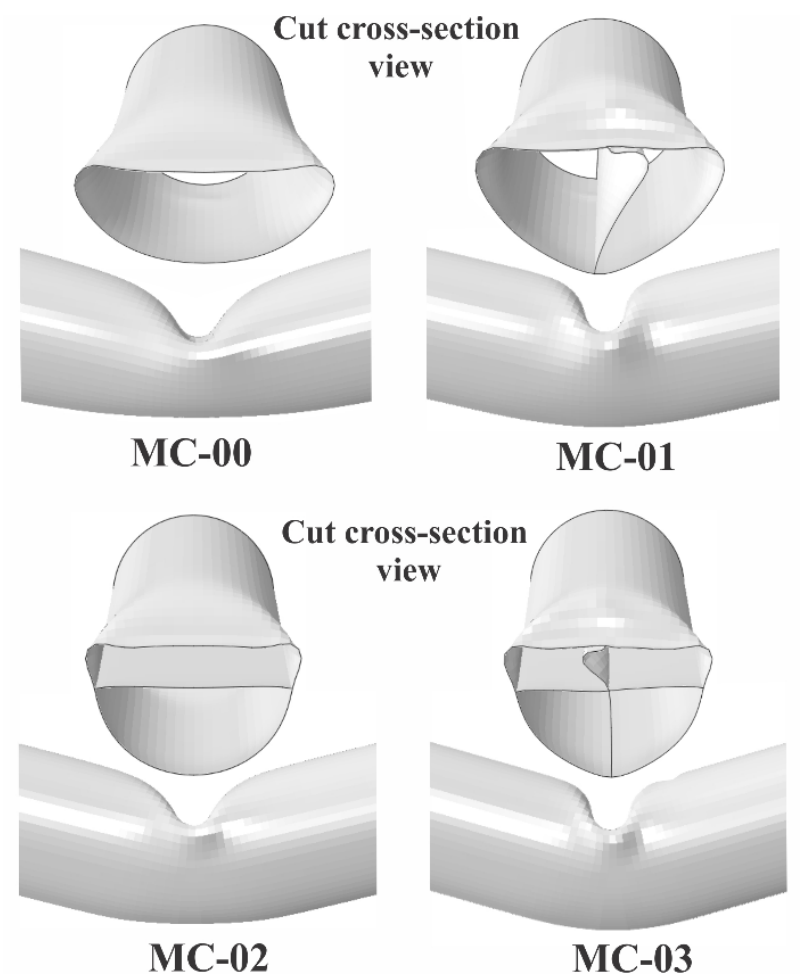

Fig. 10. Final bending deformation state for the evaluated structures I.
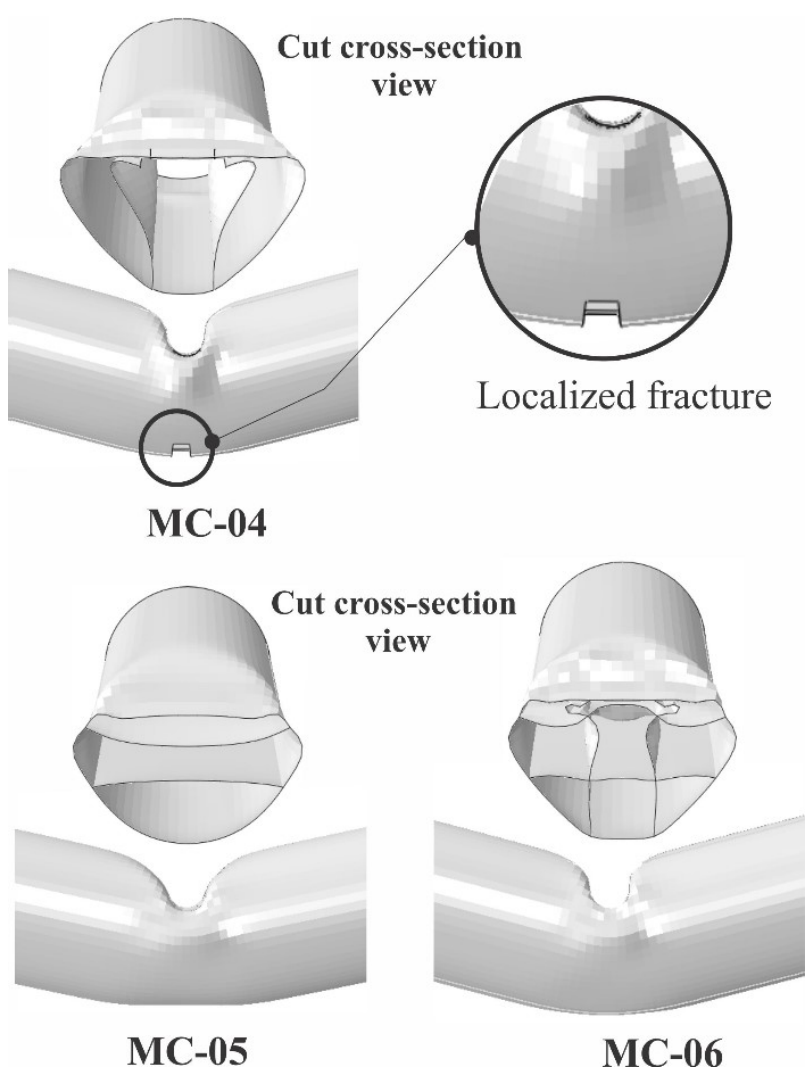

Fig. 11. Final bending deformation state for the evaluated structures II.

Since all structures have different thickness values in order to keep the mass constant (162 gr), a summary of the results obtained from numerical simulations is presented in Table 3. Contrary to what was expected, a 
decrease in thickness did not result in a low peak load $\left(\mathrm{P}_{\max }\right)$ parameter. The highest initial bending resistance to collapse corresponds to the MC-01 profile $(8.90 \mathrm{~kJ})$. Physically, an additional rib in a vertical arrangement increases the resistance to plastic rolling deformation of the circular cross-section. On other hand, energy absorption $\left(E_{a}\right)$ was determined by bending stability of the structures, deformation of the flanges profile and the number of hinge lines formed. If the MC-02 and MC-05 profiles are excluded, the remaining structures had better $\mathrm{E}_{\mathrm{a}}$ and $\mathrm{P}_{\mathrm{m}}$ performance in a range from $6 \%$ to $34 \%$. The greatest resistance to the formation of stationary and traveling hinge lines was shown by a MC-01 profile with an $E_{a}$ value of $0.201 \mathrm{~kJ}$.

Table 3. Summary of numerical results.

\begin{tabular}{ccccc}
\hline Code & $\mathbf{P}_{\max }(\mathbf{k N})$ & $\mathbf{P}_{\mathbf{m}}(\mathbf{k N})$ & $\mathbf{E}_{\mathbf{a}}(\mathbf{k J})$ & Fracture \\
\hline MC-00 & 6.55 & 4.90 & 0.148 & No \\
MC-01 & 8.90 & 6.72 & 0.201 & No \\
MC-02 & 6.22 & 4.84 & 0.145 & No \\
MC-03 & 7.08 & 5.61 & 0.168 & No \\
MC-04 & 8.10 & 6.21 & 0.186 & Yes \\
MC-05 & 6.44 & 4.84 & 0.145 & No \\
MC-06 & 7.50 & 6.40 & 0.191 & No \\
\hline
\end{tabular}

In order to establish a tendency towards energy absorption $\left(E_{a}\right)$, specific energy absorption (SEA) is presented in Figure 12. It can be seen that $E_{a}$ depends mainly on the partition plates arrangement and secondly by the number of ribs in the cross-section. Considering the single circular profile (MC-00), an improvement in $\mathrm{E}_{\mathrm{a}}$ is obtained when a partition plate is located in a vertical arrangement beneath the puncher. Opposite behaviour was obtained placing the plates horizontally (MC-02 and MC-05). The MC-01 profile obtained the maximum SEA value of 1.20 $\mathrm{J} / \mathrm{gr}$.

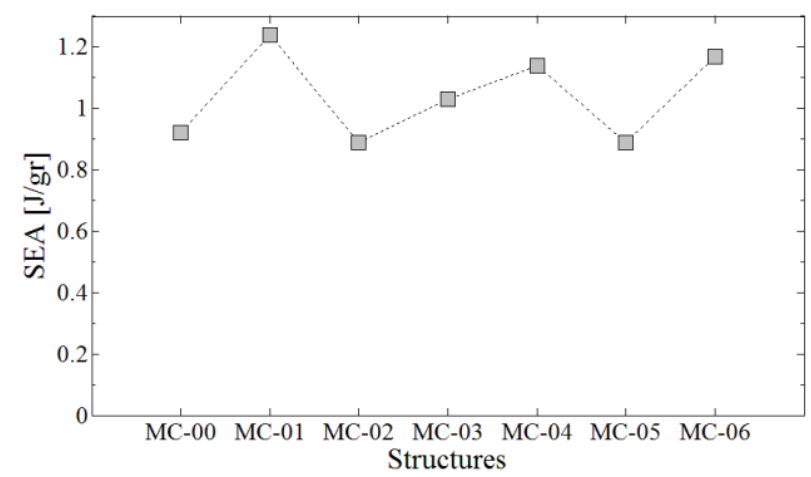

Fig. 12. Specific energy absorption [SEA].

Finally, in order to have a general understanding of the crashworthiness performance of different profiles, a comparative analysis of the crush force efficiency (CFE) is presented in Figure 13. This parameter considers the two main requirements in a crashworthiness analysis; the peak load $\left(\mathrm{P}_{\max }\right)$ and energy absorbed $\left(\mathrm{E}_{\mathrm{a}}\right)$. A value close to 1 indicates the most desirable value for energy absorbers based in thin-walled structures. For all the proposed beams, and considering the single profile as a baseline, the CFE value increased in the range $1.34 \%$ -
$16.86 \%$. The highest CFE value (0.85) was obtained by the MC-06 profile. This means a low $\mathrm{P}_{\max }$ value with a large quantity of $\mathrm{E}_{\mathrm{a}}$

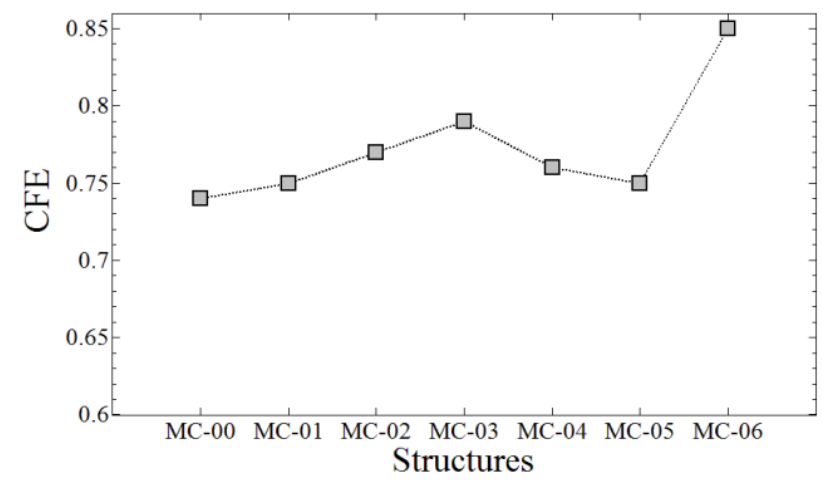

Fig. 13. Crush force efficiency for all the evaluated structures.

Finally, it was determined that a large quantity of $E_{a}$ does not guarantee the best crashworthiness performance. Likewise, in accordance with Figure 13, the addition of two partition plates to reinforce a circular cross section in the transversal and longitudinal directions allows better CFE performance. Thus, this kind of configuration could be used to reduce injuries and fatalities of car passengers during near-side impact collisions.

\section{Conclusions}

A numerical study was carried out to evaluate energy absorption of circular profiles with partition plates under lateral loads. Additionally, special emphasis was put to capture correctly the progressive failure of the structures by J-C failure model. From our study we conclude:

1. The use of partition plates in multi-cell profiles modify typical deformation state for a single profile as shown in Figure 1.

2. Considering the same mass for all structures, a decrease in thickness as the number of partition plates increases was obtained. Then, the peak load $\left(\mathrm{P}_{\max }\right)$ and energy absorption $\left(\mathrm{E}_{\mathrm{a}}\right)$ are determined by the arrangement of the ribs into the cross-section followed by the thickness value. 3. An improvement in $E_{a}$ performance $(6-34 \%)$ is obtained independently from the number of ribs, by placing partition plates in the vertical direction. However, a large value of $E_{a}$ does not ensure the best crashworthiness performance. Horizontal ribs are not recommended due to their poor $\mathrm{E}_{\mathrm{a}}$ performance (less than the $E_{a}$ value of a single profile).

4. With respect to CFE, all multi-cell profiles had better crashworthiness $(1.35-14.86 \%)$ when compared to the MC-00 single profile. Thus, a better stabilisation of the structure was obtained by including partition plates.

5 . The best crashworthiness was obtained by the MC-06 profile $(0.85)$. This allows us to conclude that better mechanical behaviour of the structures occurs when the cross-section is reinforced in the longitudinal and transversal directions. Thus, the MC-06 profile should be considered to control the lateral load in automobiles, i.e. side-door impact beam design.

6. The effectiveness of the theoretical failure evolution model was demonstrated. In this way, the failure and 
fracture of the structures was correctly captured (see Fig 11).

The authors are grateful for the financial support received from the Universidad Autonoma de Ciudad Juarez.

\section{References}

1. Organization WH. Global Status Report on Road Safety. (World Health Organization, Switzerland, 2015)

2. C. Liu, J.C. Pressley, Inj Epidemiol. 3, 17-11.(2016)

3. Q. Estrada, D. Szwedowicz, et al., Proc Inst Mech Eng Part D J Automob Eng. (to be published)

4. Z. Wang, Z. Li, X. Zhang. Thin-Walled Struct. 107, 287-299 (2016)
5. Huang Z, Zhang X, Zhang H. Thin-Walled Struct. 131, 179-191 (2018)

6. X. Zhang, H. Zhang, K. Leng. Thin-Walled Struct. 127, 728-740 (2018)

7. A. Ghadianlou, S Bin Abdullah. Thin-Walled Struct. 67, 25-33 (2013)

8. M. Elchalakani, X.L. Zhao, R.H. Grzebieta. Int J Mech Sci. 44, 1117-1143 (2002)

9. G.R. Johnson, W.H. Cook. Eng Fract Mech. 21, 3148. (1985)

10. H. Hooputra, H. Gese, H. Dell, H. Werner Int. J. Crashworthiness 9, 449-464 (2004)

11. Z. Wang, Y. Zhao, X. Liang, Z. He. Adv Mater Sci Eng. 2013, 1-13 (2013)

12. Z. Li, F. Lu. J Reinf Plast Compos. 34, 761-768 (2015) 